\title{
Respecto al consentimiento informado y su documentación
}

\author{
Aldo Barajas-Ochoa' y Zalathiel Barajas-Ochoa² \\ ${ }^{1}$ Departamento de Medicina, Rutgers New Jersey Medical School, Nueva Jersey, Estados Unidos; ${ }^{2}$ Instituto Mexicano del Seguro Social, Hospital \\ de Especialidades 25, Monterrey, Nuevo León, México
}

Leímos con interés el artículo "El consentimiento informado: recomendaciones para su documentación" escrito por Celis et al., ${ }^{1}$ publicado en el número 6 de 2018, de Gaceta Médica de México.

En el artículo se señala acertadamente que el consentimiento informado $(\mathrm{Cl})$ es un acto de igualdad que va más allá de la declaración de riesgos y beneficios. Consideramos importante insistir en la diferencia fundamental entre el $\mathrm{Cl}$ y el documento del consentimiento informado $(\mathrm{DCl})$. $\mathrm{El} \mathrm{Cl}$ es un proceso complejo que requiere cinco elementos: capacidad de decisión, explicación de la intervención propuesta, entendimiento, voluntariedad y autorización. ${ }^{2,3}$ Primero, el paciente (o su representante) debe tener capacidad de decisión. En seguida, el médico (idealmente quien realizará la intervención o quien conozca la intervención a fondo) debe asegurarse que el paciente entienda el diagnóstico y pronóstico de la enfermedad, y explicar de forma entendible la naturaleza y propósito de la intervención planteada, los riesgos y beneficios, y las alternativas disponibles. ${ }^{4}$ Finalmente, el paciente decide sin coerción y consiente o no a la intervención. El $\mathrm{Cl}$ es un proceso de comunicación $^{3}$ y el $\mathrm{DCl}$ es solo una forma de plasmar la autorización del paciente..$^{2-4}$ Que un paciente firme un $\mathrm{DCl}$ no implica la existencia del $\mathrm{Cl}^{4}{ }^{4}$

Además, en el artículo se señala que el propósito del $\mathrm{DCl}$ no es proteger al médico (este uso distorsionado está bien documentado $\left.{ }^{4,5}\right)$, "sino proteger a los pacientes de eventuales abusos y omisiones del personal de salud". 'Resulta alarmante pensar en esos "abusos" al paciente, que nos hacen cuestionar la idoneidad para ser personal de salud de quienes violan deliberadamente los principios de beneficencia y no maleficencia.

\section{Bibliografía}

1. Celis MÁ, Halabe J, Arrieta O, Burgos R, Campillo C, De la Llata M, et al. El consentimiento informado: recomendaciones para su documentación. Gac Med Mex. 2018;154:716-718. DOI: 10.24875/GMM.18004339.

2. De la Mora-Molina H, Barajas-Ochoa A, Sandoval-García L, Navarrete-Lorenzon M, Castañeda-Barragán EA, Castillo-Ortiz JD, et al. Trends of informed consent forms for industry-sponsored clinical trials in rheumatology over a 17-year period: Readability, and assessment of patients' health literacy and perceptions. Semin Arthritis Rheum. 2018;48:547552. DOI: 10.1016/j.semarthrit.2018.03.008.

3. Grady C. Enduring and emerging challenges of informed consent. N Engl J Med. 2015;372:855-862. DOI: 10.1056/NEJMra1411250.

4. Jefford M, Moore R. Improvement of informed consent and the quality of consent documents. Lancet Oncol. 2008;9:485-493. DOI: 10.1016/ S1470-2045(08)70128-1.

5. Faden R, Beauchamp T. A history and theory of informed consent. Nueva York, EE. UU.: Oxford University Press; 1986.
Gac Med Mex. 2019;155:217

Disponible en PubMed www.gacetamedicademexico.com 